\title{
CAPACIDADE DE NODULAÇÃO DE CEM LEGUMINOSAS DA AMAZÔNIA.
}

\author{
Luiz A. G. de SOUZA ${ }^{1}$, Marlene F. da SILVA ${ }^{2}$, Francisco W. MOREIRA ${ }^{1}$
}

\begin{abstract}
RESUMO - Neste trabalho são apresentadas informações sobre a habilidade nodulífera de 100 espécies da família Leguminosae, nativas dos estados do Amazonas, Pará, Rondônia e Roraima (Brasil), e avaliadas em seus habitats de origem e/ou em viveiro. As observações foram efetuadas em mata primária, várzea, campinarana, igapó, savana, floresta secundária, etc., totalizando II habitats visitados. Foram também, efetuados estudos de comportamento de mudas em viveiro, em diferentes solos da regiāo. A identificação das espécies foi processada no herbário do INPA. Foi constatado que $63 \%$ das espécies foram capazes de nodular, sendo esta característica detectada em solos ácidos com diferentes texturas e fertilidades, com $\mathrm{pH}$ variando entre 3.9 e 5.9 . A nodulação foi mais frequente em espécies das sub-famílias Mimosoideae e Faboideae do que em Caesalpinioideae. Os nódulos encontrados apresentaram uma grande variabilidade de cor e formas, predominando os coralóides de cor creme. Foi observada pela primeira vez a nodulação em 21 espécies, e dentre elas 2 novos gêneros são apresentados como nodulíferos: Acosmium (Faboideae) e Zollernia (Caesalpinioideae). são apresentadas também, novas descrições de 11 espécies que não apresentaram nódulos. $\mathrm{O}$ habitat, hábito de crescimento da planta, textura e fertilidade do solo, não interferiram na ocorrência da nodulação que foi relacionada a outros fatores como a propriedade intrínseca das espécies, e ocorrência de estirpes de rizóbio compatíveis em cada local.
\end{abstract}

Palavras-Chave: Leguminosas Nativas, Nodulação, Fixação de Nitrogênio.

Capacity of Nodulation of one hundred Leguminosae of Amazônia.

\begin{abstract}
This paper includes informations about nodulation capacity of 100 native species of the legume family from the states of Amazonas, Pará, Rondônia and Roraima (Brasil), avaluated in their original habitats and/or in nursery plots. Field observations were made in "terra-firme" forests, várzea, campinarana, savana, igapó, secondary forest, etc. Plant material was collected in a total of 11 habitats. The species were studied in nursery beds using soils from diferent regions. The genera and species of plants were identified at the INPA herbarium. The survey verified that $63 \%$ of the species presented nodules. These characteristics were observed in acid soils with different textures and fertility levels, with a pH varying from 3.9 to 5.9. Nodulation was more frequent in species of the sub-families Mimosoideae and Faboideae, than in Caesalpinioideae. The nodules collected had a great variability in color and shape, being more common coral shapes and creamy color, Data for nodulation of 21 legume species included 2 new genera not reported before: Acosmium (Faboideae) and Zollernia (Caesalpinioideae). Eleven species, which were tested for the first time, did not show nodulation. Habitat, type of plant growth, soil texture and low fertility had no effect on nodulation, which is considered to be related with other factors like properties inherent to the species and occurrence of compatible rhizobium strains in the different sites.
\end{abstract}

\section{INTRODUÇÃO}

Na vegetação amazônica, as leguminosas têm amplo destaque em diferentes ecossistemas, sendo a mais importante família de plantas dentre as lenhosas e a mais numerosa em espécies e gêneros nativos (DUCKE,

I INPA - Instituto Nacional de Pesquisas da Amazônia /CPCA, Caixa Postal 478, 9011-970 Manaus, AM, Brasil.

${ }^{2}$ Instituto de Tecnologia da Amazônia - UTAM/CEPEF, Av. Darcy Vargas 1,200, 9055-028, Manaus, AM, Brasil. 
1949; DUCKE \& BLACK, 1954; SILVA et al., 1989). Entretanto, a maioria das leguminosas da região ainda não foram estudadas quanto à sua capacidade de nodular e fixar nitrogênio. Identificar esta condição em essências nativas é uma das prioridades da pesquisa com fịxação simbiótica de nitrogênio.

As espécies nodulíferas representam um material genético importante para o balanço de nitrogênio nos trópicos, que ocorre através da liberação deste elemento para o ecossistema, ou para culturas associadas, pela decomposição de nódulos e matéria orgânica com baixa relação Carbono/Nitrogênio (RUSSO, 1983). Em solos deficientes de nitrogênio, as leguminosas fixadoras competem com vantagem com espécies não nodulíferas ou não leguminosas e são uma alternativa importante e econômica para adicionar o nitrogênio ao sistema solo-plantaanimal.

O desempenho das leguminosas nativas pode ser superior à cultivos exóticos tradicionais. Em solo Podzólico Vermelho Amarelo, MAGALHÃES (1983) verificou que o Eucalyptus teve crescimento inferior ao da "cedrorana" (Cedrelinga catenaeformis Ducke), uma mimosoídea arbórea, nodulífera. Leguminosas nativas podem ter múltiplos usos, tais como: madeira, tanino, lenha, carvão, celulose e papel, flora apícola, grãos, forragem, perfumaria, produção de óleo-resina, essências medicinais, cercasvivas, postes, ornamentais, etc. (LOUREIRO et al., 1979; NAS, 1979; SUDAM/IPT, 1981).
Estudos sobre a capacidade de nodulação e fixação simbiótica de nitrogênio na Amazônia foram efetuados por NORRIS, 1969; SYLVESTER-BRADLEY et al, 1980; MAGALHÃES et al., 1982; MATOS, 1986; MAGALHÃES \& SILVA, 1986/87 e MOREIRA et al., 1992. Nestes trabalhos as espécies examinadas eram oriundas da regeneração natural, de plantas jovens, árvores adultas ou de mudas conduzidas em viveiro.

Com o objetivo de verificar e caracterizar a nodulação em leguminosas nativas amazônicas e de descrever aspectos de sua ocorrência e ecologia, efetuou-se o presente trabalho, envolvendo informações sobre 100 espécies coletadas na região norte do Brasil.

\section{MATERIAL E MÉTODOS}

Foram estudadas 100 espécies nativas coletadas através de várias expedições de estudo em reservas ecológicas e florestais. As observações sobre a nodulação das espécies foram efetuadas em condições naturais ou em viveiro. As observações "in loco" foram realizadas nos anos de 1984, e, 1987 a 1989, nos estados do Amazonas, Pará, Rondônia e Roraima. Foram visitados os seguintes habitats: mata primária, mata secundária, campo, campinarana, savana, várzea, vazante, igapó, mata ribeirinha, e consideradas algumas espécies utilizadas na arborização urbana ou cultivadas. Na maioria das vezes, percorreram-se trilhas, margens de 
rios, beiras de estradas e áreas abertas, identificando-se as leguminosas ocorrentes em cada habitat. Para cada espécie selecionada, as raízes foram vasculhadas a partir da base do tronco, partindo-se das primárias para as secundárias. Quando não eram encontrados nódulos, novas tentativas foram efetuadas em outros indivíduos da mesma espécie, em árvores jovens ou mudas da regeneração natural.

Em todos os habitats, foram coletadas, aleatoriamente, amostra's de solo, na profundidade de até $15 \mathrm{~cm}$, para análise química e de textura. Estas análises foram efetuadas segundo o Método de Análise de Solo do Serviço Nacional de Levantamento e Conservação de Solos (EMBRAPA, 1979), no laboratório de solos da EMBRAPA, UEPAE de Manaus, AM.

Quando existentes, foram coletadas sementes para estudos complementares com mudas conduzidas em viveiro. Exsicatas botânicas foram preparadas para cada espécie listada, afim de se processar a identificação e, um exemplar de cada uma delas, quando possível, foi registrado e incorporado ao herbário do INPA. Os ensaios experimentais em viveiro foram conduzidos nas dependências do INPA/CPCA em Manaus, nos anos de1987 a1989. As sementes foram semeadas em areia, e as plântulas foram repicadas em diferentes tipos de solos, sendo em alguns casos, submetidas à inoculação com estirpes da coleção de rizóbios do INPA, e avaliadas, após o desenvolvimento.
Os nódulos coletados foram descritos quanto à forma, cor e frequencia da nodulação, e, armazenados em tubos com silica-gel, até $\mathrm{o}$ isolamento. As estirpes isoladas foram incorporadas à coleção de rizóbios da Seção de Microbiologia do Solo, CPCA/INPA.

\section{RESULTADOS E DISCUSSÃO}

Informações sobre a capacidade de nodular das espécies estudadas encontram-se na Tabela 1. Das 100 espécies listadas, $63 \%$ mostraram-se capazes de formar nódulos radiculares.

Foram coletadas espécies de 55 gêneros, sendo 38 deles nodulíferos. Nos gêneros Acacia, Cassia, Inga e Mimosa, foram observadas espécies com nódulos e outras que não apresentaram nodulação. Os nódulos foram observados em $96.9 \%$ das Fabaceas, $66.7 \%$ das Mimosaceas e em apenas $31.6 \%$ das Cesalpinaceas. Estes resultados concordam com outros autores que encontraram maior frequência de nodulação na sequência FABOIDEAE $>$ MIMOSOIDEAE $>$ CAESALPINIOIDEAE (SYLVESTERBRADLEY et al., 1980; ALLEN \& ALLEN, 1981; FARIA et al., 1984).

Em todos os habitats estudados, haviam espécies capazes de estabelecer nodulação com rizóbios, sendo que em campinarana, savana, campo e vazante, todas as espécies coletadas tinham essa propriedade (Tab.1). Em condições naturais, os nódulos se instalam normalmente em raízes secundárias que podem estar próximas à base do tronco ou a alguns metros de distância dele. Em 


\begin{tabular}{|c|c|c|c|c|c|c|c|c|c|}
\hline \multirow{2}{*}{ ESPECIES } & \multirow{2}{*}{$\begin{array}{l}\text { LOCAL } \\
\text { DE } \\
\text { COLETA }\end{array}$} & \multirow{2}{*}{ HABITAT } & \multirow{2}{*}{ PORTE } & \multirow{2}{*}{$\begin{array}{l}\text { TEXTURA } \\
\text { DOLO } \\
\text { SOLO }\end{array}$} & \multicolumn{2}{|c|}{$\begin{array}{l}\text { CONSYATACAOODE } \\
\text { NODULACAO CC }\end{array}$} & \multicolumn{3}{|c|}{ CARACTERISTICAS DOS NODULOS } \\
\hline & & & & & CAMPO & VIVEIRO & FORMA & COR & INTENDIDADE \\
\hline \multicolumn{10}{|l|}{ CAESALPINIOIDEAE } \\
\hline Apuleia leiocama (Vog.) Mcbr & PA & 6 & ÁRVORE & ARGILOSO & NĀO & NAO & - & - & - \\
\hline Bauhinia acreana Harms. & RO & 8 & ÁRVORE & ARGRoso & NĀOO & NÃO & - & - & - \\
\hline Bauhinia angularis Harms. **. & AM & 8 & ARBUSTO & ARGROSO & NĀO & NĀO & $\cdot$ & - & $=$ \\
\hline & & 2 & $\begin{array}{l}\text { ARBUSTO } \\
\text { ARVORE }\end{array}$ & $\begin{array}{l}\text { ARGILOSO } \\
\text { ARGLOSO }\end{array}$ & NĀO & NĀO & - & $\cdot$ & - \\
\hline $\begin{array}{l}\text { Bauhinia purpurea DC ex Walp. } \cdots \\
\text { Bauhinia ungulata L. ... }\end{array}$ & $\begin{array}{l}\text { AM } \\
\text { RR }\end{array}$ & $\begin{array}{l}1 \\
8\end{array}$ & ARBUSTO & $\begin{array}{l}\text { ARGILOSO } \\
\text { ARGILOSO }\end{array}$ & $\begin{array}{l}\text { ND } \\
\text { NÃO }\end{array}$ & $\begin{array}{l}\text { NÃO } \\
\text { NÃO }\end{array}$ & $\therefore$ & - & $\dot{-}$ \\
\hline Caesalpinia ferrea Mart. ex Tul. & RO & 2 & ÁRVORE & ARGILOSO & NĀO & NÁO & - & . & 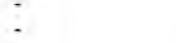 \\
\hline Cassia fistula $\mathrm{L}$ & AM & 1 & ÁRVORE & ARGILO-ARENOSO & ND & NÃO & $\cdot$ & . & - \\
\hline Cassia grandis L. $f$. & AM & 8 & ÁRVORE & ARENOSO & ND & NÄO & 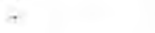 & - & . \\
\hline Cassia leiandra Bènth. & AM & 10 & ARVORE & SILTOSO & NĀO & NÃO & s & - & - \\
\hline Cassia lucens vog. $\cdots$ & AM & 8 & ÁRVORE & ARGLOSO & NÃO & NĀO & $\cdot$ & - & - \\
\hline Cassia mimosoides 2 . $\cdots$ & $\mathbf{R R}$ & 9 & ERVA & ARGILO-ARENOSO & $\operatorname{sim}$ & $\operatorname{SIM}$ & ESFÉRICA & BRANCA & VARIÁVEL \\
\hline Cassia moschata H.B.K & $\mathbf{R R}$ & 7 & ÁRVORE & SILTOSO & NĀO & NĀO & - & - & - \\
\hline Cassia negrensis Irwin. & AM & $\mathbf{s}$ & ÁRVORE & SUTOSO & ND & SIM & CORAL & MARRON & VARTÄVEL \\
\hline Chamaecrista nictitans (L.) Moench. & RO & 4 & ERVA & ARGILOSO & $\sin$ & $\operatorname{siM}$ & ESFÉRICA & CREME - & ABUNDANTE \\
\hline $\begin{array}{l}\text { Crudia amazonica Spruce ex Benth. } \\
\text { Cynometra spruceana var }\end{array}$ & AM & 5 & ÁR VORE & SILTOSO & NĀO & NÃO & - & - & - \\
\hline $\begin{array}{l}\text { Cynometra spruceana var } \\
\text { phaseolocarpa (Hayne) Dwyer. } \cdots\end{array}$ & AM & 5 & ÁRVORE & SILTOSO & ND & NÃO & - & - & - \\
\hline Dialium guianense (Aubl) Sandw. & RR & 6 & ÁRVORE & ARENOSO & NÃO & NĀO & $\cdot$ & - & - \\
\hline Elizabetha coccinea Benth *. & RR & 7 & ARVORE & SILTOSO & $\operatorname{SIM}$ & NĀO & ESFÉRICA & CREME & VARIÁVEL \\
\hline Eperua duckeana Cowan. ... & AM & 6 & ÁRVORE & ARGILOSO & NĀO & NĀO & - & - & - \\
\hline Eperua glabriflore (Ducke) Cowan. ... & AM & 6 & ÁR VORE & ARGILOSO & NĀO & NĀO & - & - & - \\
\hline Hymenaca courbaril $\mathrm{L}$. & PA & 6 & ÁRVORE & ARGROSO & $\mathrm{NABO}$ & NÃO & - & . & . \\
\hline Hymenaca parvifolia Hubet. & $\mathbf{R R}$ & 6 & ÁRVORE & ARENOSO & NĀO & NĀO & . & . & $=$ \\
\hline Macrolobium acaciifolium (Benth) Benth & AM & 5 & ARVORE & SILTÓSO & NĀO & NÁO & - & - & - \\
\hline Mora paraensis Ducke. ... & AM & 5 & ÁRVORE & SILTOSO & NÄO & NĀO & . & - & - \\
\hline Peltogyne gracilipes Ducke. $\cdots$ & $R R$ & 6 & ÁRVORE & ARENOSO & NÁO & NĀO & - & - & - \\
\hline $\begin{array}{l}\text { Peliogyne paniculata ssp } \\
\text { pubescens (Benth) MF da Silva. }\end{array}$ & $\mathbf{R R}$ & 6 & ÁRVORE & ARGILO-ARENOSO & NĂOO & NẢO & - & - & - \\
\hline $\begin{array}{l}\text { Peltogyne venosa ssp densifora } \\
\text { (Spruce ex Benth) MF da Silva. }\end{array}$ & AM & 5 & ÁRVORE & SILTOSO & NĀO & NĀO & - & - & - \\
\hline Senna multijuga (LC Rich) Irwin \& Barneby & RO & 8 & ÄRVORE. & ARGROSO & NĀO & NÃO & $-\quad$ & $-\quad a_{0}$ & 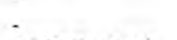 \\
\hline Sclerolobium chrysophyllum Poepp \& Endl... & RR & 9 & ÁRVORE & ARENOSO & $\operatorname{sim}$ & ND & CORAL & MARRON & ABUNDANTE \\
\hline Sclerolobium paraensis Huber. $\cdots$ & AM & 6 & ÁRVORE & ARGILO-ARENOSO & $\operatorname{sim}$ & ND & ALONGADA & LARANIA & ABUNDANTE \\
\hline Swartzia laevicarpa Amshoff. & AM & $s$ & ÁRVORE & SILTOSO & ND & SIM & CORAL & CREME & ABUNDANTE \\
\hline Swartzia laurifolia Benth. .. & RR & 6 & ÁRVORE & ARENOSO & SIM & ND & ALONGADA & CREME & ABUNDANTE \\
\hline Swartzia laxifora Bong ex Benth. ** & RR & 9 & ARVORE & ARENOSO & SIM & $\operatorname{sim}$ & CORAL & MARRON & ABUNDANTE \\
\hline Swarizia polyphylla DC. & AM & 7 & ARVORE & ARGILOSO & SIM & SIM & CORAL & LARANJA & ABUNDANTE \\
\hline Tachigalia paniculata Aubl. & AM & 5 & ÁRVORE & SILTOSO & $\sin$ & SIM & ESFERICA & MARRON & ABUNDANTE \\
\hline Zollemia paraensis Huber. - & $R R$ & 7 & ARVORE & SILTOSO & SIM & ND & ALONGADA & MARRON & ABUNDANTE \\
\hline
\end{tabular}


TABELA 1 - Ciontinuação.

ESPECIES

LOCAL
COLETA HABTAT PORTE

TEXTURA
DOLO

CONSTATACÃO DE

CARACTERISTICAS DOS NODULOS

\section{FABOIDEAE}

Acosmium nitens (Vog.) Yakovl.

Andira parviflora Ducke. "*

Batesia floribunda Spr. ex Bench. *..

Centrolobium paraense Tul

Centrosema brasilianum (L.) Benth

Clitoria amazonum (Mart) Benth.

Clitoria fairchildiana Howard

Coursetia ferruginea (Kunt) Lavin...

Dalbergia riedelii (Benth) Sandw. **

Desmodium adscendens (L.) DC

Desmodium barbatum (L.) Bentri

Dioclea bicolor Benth

Dioclea glabra Mart ex Benth.

Dioclea virgata (L.C. Rich) Amshoff.

Flemingia macrophylla (Willd) Merr.

Galactia iussiaeana Kunth. *

Hymenolobium petraeum Ducke. **

Indigofera lespedesioides H,B.K. *.

Machaerium foribundum Benth. *.

Machaerium inundatum (Benth) Ducke

Mucuna altissima (Jacks) DC

Ormosia coarctata Jacks. *

Ormosia costulata (Miq.) Kleinh. *.

Ormosia discolor Spruce ex. Benth. ..

Ormosia excelsa Spruce ex. Benth.

Ormosia flava (Ducke) Rudd. *.

Ormosia macrocalyx Ducke.

Ormosia paraensis Ducke. *

Phaseolus pilosus H.B.K. *

Platymiscium duckei Huber

Platymiscium paraense Huber. **

Rhynchosia minima (L.) DC.

\begin{tabular}{|c|c|c|c|c|c|c|c|c|}
\hline$A M$ & 7 & ÁRVORE & SILTOSO & $\operatorname{sim}$ & $\operatorname{sim}$ & CORAL & AMARELO & ABUNDANTE \\
\hline $\mathrm{AM}$ & 6 & ÁRVORE & ARGILOSO & NĀO & SIM & ALONGADA & PRETO & BADXA \\
\hline$A M$ & 6 & ÁRVORE & ARENOSO & NĀO & NĀO & - & - & - \\
\hline$R R$ & 6 & ÁRVORE & ARGILO-ARENOSO & NĀO & SIM & ESFÉRICA & MARRON & VARLAVEL \\
\hline AM & 4 & ERVA & ARGILO-ARENOSO & $\operatorname{SIM}$ & SIM & ESFÉRICA & BRANCO & ABUNDANTE \\
\hline AM & 5 & ARBUSTO & SEDIMENTAR & SIM & $\operatorname{sim}$ & ESFÉRTCA & CREME & ABUNDANTE \\
\hline AM & 1 & ÁRVORE & ARENOSO & SIM & $\operatorname{sim}$ & ESFÉRICA & BRANCO & ABUNDANTE \\
\hline RR & 6 & ARBUSTO & ARENOSO & NĀO & NĀo & - & - & - \\
\hline RR & 6 & CIPO & ARENOSO & SIM & ND & ALONGADA & CREME & VARLÁVEL \\
\hline AM & 8 & ERVA & ARGILOSO & stM & SIM & ESFÉRICA & CREME & ABUNDANTE \\
\hline RR & 9 & ERVA & ARENOSO & SIM & $\sin$ & ESFERICA & MARRON & ABUNDANTE \\
\hline AM & 5 & CrPO & SILTOSO & SIM & SIM & CORAL & BRANCO & ABUNDANTE \\
\hline AM & 8 & CIPO & ARGILOSO & SIM & ND & CORAL & VERMELHO & VARIÁVEL \\
\hline RR & 11 & ERVA & ARGILOSO & $\operatorname{sim}$ & ND & ALONGADA & BRANCO & BALXA \\
\hline AM & 2 & ARBUSTO & ARGILOSO & SIM & SIM & ESFERICA & CREME & ABUNDANTE \\
\hline RR & 9 & ERVA & ARENOSO & $\sin$ & $\operatorname{sim}$ & ESFERICA & CREME & ABUNDANTE \\
\hline RR & 6 & ÁRVORE & ARENOSO & NĀO & SIM & ESFÉRICA & CREME & VARLÁVEL \\
\hline RR & 8 & ERVA & ARGILOSO & SIM & $\operatorname{sim}$ & ALONGADA & MARRON & ABUNDANTE \\
\hline RR & 7 & ARBUSTO & SILTOSO & $\operatorname{sim}$ & ND & ESFERICA & CREME & ABUNDANTE \\
\hline RR & 9 & ARBUSTO & ARENOSO & $\sin$ & NĀO & ALONGADA & MARRON & VARLÁVEL \\
\hline AM & 5 & CIPO & SILTOSO & ND & SIM & CORAL & PRETO & ABUNDANTE \\
\hline RR & 9 & ÁRVORE & ARENOSO & SIM & $\operatorname{sim}$ & CORAL & CREME & VARIÁVEL \\
\hline AM & 3 & ÁRVORE & ARENOSO & SIM & $\operatorname{sim}$ & CORAL & AMARELO & ABUNDANTE \\
\hline AM & 8 & ÁRVORE & ARGILO-ARENOSO & SIM & SIM & CORAL & CREME & VARLÁVEL \\
\hline AM & 5 & ÄRVORE & SILTOSO & ND & $\operatorname{sim}$ & CORAL & CREME & VARĹVELL \\
\hline RR & 6 & ÁRVORE & ARGILO-ARENOSO & NĀO & $\operatorname{sim}$ & CORAL & AMARELO & BADXA \\
\hline AM & $s$ & ÁRVORE & SILTOSO & ND & SIM & CORAL & CREME & ABUNDANTE \\
\hline AM & 8 & ÁRVORE & ARENOSO & SIM & sim & CORAL & CREME & ABUNDANTE \\
\hline RR & 11 & ERVA & ARGLOOSO & SIM & $\operatorname{sim}$ & ESFERTICA & CREME & ABUNDANTE \\
\hline AM & 2 & ÁRVORE & ARGILOSO & ND & SIM & ESFÉRICA & CREME & VARLÁVEL \\
\hline RR & 6 & ÁRVORE & ARGILOSO & sim & ND & CORAL & MARRON & ABUNDANTE \\
\hline AM & 10 & ERVA & SuLtoso & $\sin$ & SIM & ALONGADA & CREME & ABUNDANTE \\
\hline AM & 4 & ERVA & ARENOSO & $\operatorname{SIM}$ & SIM & ESFERICA & BRANCO & ABUNDANTE \\
\hline
\end{tabular}




\begin{tabular}{|c|c|c|c|c|c|c|c|c|c|}
\hline \multirow{2}{*}{ ESPECIES } & \multirow{2}{*}{$\begin{array}{l}\text { LOCAL } \\
\text { DE } \\
\text { COLETA }\end{array}$} & \multirow{2}{*}{ HABITAT } & \multirow{2}{*}{ PORTE } & \multirow{2}{*}{$\begin{array}{l}\text { TEXTURA } \\
\text { DO } \\
\text { SOLO }\end{array}$} & \multicolumn{2}{|c|}{$\begin{array}{l}\text { CONSTATACAOODE } \\
\text { NODULACAO } \cdot \mathrm{C}\end{array}$} & \multicolumn{3}{|c|}{ CARACTERISTICAS DOS NODULOS } \\
\hline & & & & & CAMPO & VIVEIRO & FORMA & COR & INTENDIDADE \\
\hline MIMOSOIDEAE & & & & & & & & (f) & \\
\hline Acacia multipinnata Ducke. & RR & 7 & ARBUSTO & SILTOSO & $\sin$ & ND & ALONGADA & MARRON & BADXA \\
\hline Acacia polyphylla DC. & RO & 6 & ÁRVORE & ARGROSO & NÃO & NĀO & - & $-\quad x$ & - \\
\hline Adenanthera pavonina $L$ & AM & I & ÁRVORE & ARGILOSO & NĀO & NẢO & $=$ & . & - \\
\hline Albizia corymbosa (L.C. Rich) GP Lewis ** & RR & 7 & ÁRVORE & SILTOSO & SIM & $\sin$ & CORAL & BRANCA & ABUNDANTE \\
\hline Calliandra surinamensis Benth. & AM & 1 & ARBUSTO & ARGILOSO & $\operatorname{sim}$ & SIM & ESFERICA & CREME & ABUNDANTE \\
\hline Cedrelinga catenaeformis Ducke. & AM & 2 & ÁRVORE & ARGILOSO & $\operatorname{sim}$ & $\sin$ & ESFERICA & VERMELHO & ABUNDANTE \\
\hline Dinizia excelsa Ducke. & PA & 6 & ÁRVORE & ARGILOSO & NẢO & NĀO & 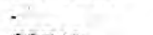 & - & 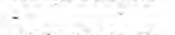 \\
\hline Entada polyphylla Benth. & AM & 3 & CIPÓ & ARENOSO & NĀO & $\operatorname{sim}$ & CORAL & LARANJA & ABUNDANTE \\
\hline Entada polystachya (L.) DC. ** & $\mathbf{R R}$ & 8 & CIPO & ARGROSO & NÃO & $\sin$ & CORAL & LARANJA & ABUNDANTE \\
\hline Enterolobium ciclocarpum (Jacq.) Griseb. & RR & 6 & ÁRVORE & ARGILO-ARENOSO & $\operatorname{siM}$ & SIM & CORAL & BRANCO & ABUNDANTE \\
\hline Enterolobium schomburgkii Benth. & RR & 6 & ÁRVORE & ARENOSO & $\operatorname{sim}$ & $\operatorname{sim}$ & ESFÉRICA & CREME & VARIÁVEL \\
\hline Inga' jlba (Sw.) Willd. & AM & 8 & ARVORE & ARGILO-ARENOSO & NĀO & ND & 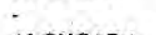 & $\cdot$ & - \\
\hline Inga disticha Benth. ** & AM & 6 & ÁRVORE & ARGILO-ARENOSO & SIM & $\operatorname{sim}$ & ALONGADA & BRANCA & VARLÁVEL \\
\hline Inga edulis Mart. & AM & 8 & ÁRVORE & ARGILOSO & SIM & SIM & ESFERICA & BRANCA & ABUNDANTE \\
\hline Inga myriantha Poepp. ... & $\mathbf{R R}$ & 6 & ÁRVORE & ARGILO-ARENOSO & NÃO & ND & $\cdot$ & - & - \\
\hline Inga nobilis Willd. ** & RR & 7 & ÁRVORE & SILTOSO & $\sin$ & $\operatorname{sim}$ & ESFERICA & CREME & ABUNDANTE \\
\hline Inga thibaudiana DC, *" & $A M$ & 8 & ÁR VORE & ARGILOSO & NÃO & $\operatorname{sim}$ & ESFERICA & CREME & ABUNDANTE \\
\hline Mimosa debilis H.B.K. *- & RO & 8 & ARBUSTO & ARENOSO & SIM & ND & ESFERICA & BRANCA & ABUNDANTE \\
\hline Mimosa pudica L. & RR & 9 & ERVA & ARGILOSO & $\sin$ & $\operatorname{siM}$ & CORAL & MARRON & ABUNDANTE \\
\hline Mimosa spruceana Benth. & AM & 8 & CIPÓ & ARGILOSO & NĀO & NĀO & - & $\cdot$ & - \\
\hline Parkia decussata Ducke. & AM & 8 & ÁRVORE & ARGILOSO & NĀO & NĀO & - & $\cdot$ & $\cdot$ \\
\hline Parkia discolor Spr, ex Benth. .... & AM & 7 & ÁRVORE & SILTOSO & NÃO & NĀO & $\cdot$ & - & - \\
\hline Parkia nitída Miguel. & AM & 8 & ÁRVORE & ARGILOSO & NÄO & NÃO & $\cdot$ & - & r \\
\hline Parkia pendula (Willd.) Benth ex Walpers & PA & 6 & ÁRVORE & ARGILOSO & NÄO & NĀO & - & isongen & - PSTMATr \\
\hline Pentaclethra macroloba $\mathrm{O}$. Kuntze. & AM & 5 & ÁRVORE & SILTOSO & $\sin$. & $\sin$ & CORAL & MARRON & ABUNDANTE \\
\hline Pithecellobium arenarium Ducke. & AM & 3 & ÁRVORE & ARENOSO & SIM & $\operatorname{sim}$ & ESFERICA & LARANJA & ABUNDANTE \\
\hline Pithecellobium jupumba (Willd.) Urb. & $\mathbf{R R}$ & 6 & ÁRVORE & ARENOSO & SIM & $\operatorname{sim}$ & ALONGADA & CREME & ABUNDANTE \\
\hline Pithecellobium saman (Jacq.) Benth. & RR & 6 & ÁRVORE & ARENOSO & $\operatorname{sim}$ & $\operatorname{siM}$ & CORAL & CREME & ABUNDANTE \\
\hline Sthryphnodendron guianensis (Aubl.) Benth. & AM & 8 & ÁRVORE & ARGILO-ARENOSO & $\operatorname{sim}$ & $\sin$ & CORAL & LARANJA & VARIÁVEL \\
\hline Zygia cauliflorum (Willd.) Killip. & AM & 5 & ÁRVORE & SILTOSO & SIM & SIM & ALONGADA & CREME & VARLÁVEL \\
\hline
\end{tabular}

*A - Novas descriçōes na literatura para: $\bullet$ - Gênero nodulifero; $* *$. Especie nodulífera; *** - Especie năo nodulífera.

B - Habitat: 1 - Artrorizaçăo urbana; 2 - Área cultivada; 3 - Campinarana: 4-Campo; 5 - Igaps; 6 - Mata primária; 7 - Mata ribeirinha; 8 - Mata secundária; 9 - Savana; 10 - Várzea; 11 - Vazante.

- D - Quando a nodulação foi observada com nódulos esféricos, alongados e coralóides na mesma planta esta foi descrita como coral; Quando os nódulos eram esféricos e alongados, foram descritos

•E - Para intensidade considerou-se: Baixa - menos de 10 nódulos por planta; Variável - quando haviam irregularidades na nodulação ou esta se encontrava de i0-20 nódulos por planta: Abundante mais que 20 nódulos por planta. 
campinarana, constatou-se farta nodulação coralóide nas raízes secundárias de Ormosia costulata e Pithecellobium arenarium, concentradas à 3 metros do tronco das árvores adultas comprovando as observações feitas por SYLVESTER-BRADLEY et al.(1980) para este ambiente. Um fato interessante a considerar é que a vegetação de campinarana apresenta condições desfavoráveis para a colonização de plantas, devido aos fatores ambientais críticos, tais como: baixa fertilidade, altas temperaturas no solo, drenagem rápida e acidez elevada (PRANCE, 1975).

A textura do solo não limitou o estabelecimento da nodulação (Tab1). Em solos argilosos, espécies como Inga edulis e Platymiscium paraensis podem apresentar nodulação abundante. Algumas espécies observadas sem nódulos nesses solos, geralmente em mata primária, nodularam posteriormente em viveiro. Foram observados nódulos em $3 /$ 4 das espécies coletadas em solos arenosos, porém, a textura do solo não parece ser responsável por essa alta ocorrência. Um fator a considerar é a disponibilidade de nitrogênio em cada sítio, uma vez que a alta permeabilidade dos solos arenosos facilita a lixiviação de nitrato, permitindo o estabelecimento da nodulação em situações de deficiência de nitrogênio.

Com relação ao porte, as árvores representaram $67 \%$ das espécies amostradas (Tab.1), e destas, 53\% foram capazes de nodular. Todos os arbustos e ervas coletadas nodularam, o mesmo ocorrendo com $86 \%$ dos cipós. DUCKE (1949), destacou a grande abundância de cipós lenhosos na Amazônia, não comparável a outras regiões brasileiras. Os cipós fixadores podem desempenhar papel ecológico na ciclagem de nutrientes da floresta: têm ciclo de vida menor que o das árvores, fácil decomposição e farta produção de folhagem, rica em nitrogênio. Embora nem todas as leguminosas arbóreas nodulem, este fenômeno é comum e sugere uma maior utilização dos benefícios da fixação de nitrogênio nos sistemas produtivos perenes da Amazônia.

Os nódulos mais encontrados foram do tipo coralóides (Tab.1), havendo também ocorrência de nódulos esféricos e alongados. Geralmente os nódulos novos eram esféricos e de cor clara, adquirindo com o tempo sua forma adulta e cor característica. A cor creme foi a mais frequente.

Foi constatada a presença de nódulos em plantas provenientes de solos ácidos e de baixa fertilidade (Tab. 2). $\mathrm{Na}$ vegetação de campinarana, nódulos foram encontrados a pH 3.9, e em áreas cultivadas este chegou a 5.9. Os solos, em geral, apresentaram baixa disponibilidade de nutrientes, exceto nas várzeas onde foram constatados maiores teores de bases trocáveis. Em vários solos, mesmo com teores excessivos de alumínio, foi constatada a ocorrência de nodulação. Teores tóxicos de alumínio no solo estão relacionados com um atrazo da nodulação, mas não impedem o estabelecimento de nódulos (CARVALHO et al., 1982).

Algumas espécies apresentaram 
grande potencial para fixação de nitrogênio por apresentarem rápido crescimento e boa nodulação em diferentes condições de solo: Clitoria fairchildiana, Enterolobium cyclocarpum, Ormosia macrocalyx, Pentaclethra macroloba, Inga edulis e Swartzia laevicarpa. Dentre as espécies com baixa nodulação, Andira parvifolia, Hymenolobium petraeum e Acacia multipinnata merecem novos estudos devido a importância

Tabela 2. Composição química dos solos coletados em diferentes habitats amazônicos.

\begin{tabular}{|c|c|c|c|c|c|c|c|}
\hline & pH & $\mathbf{P}$ & $\mathbf{K}$ & $\mathrm{Ca}$ & Mg & Al & c \\
\hline HABITAT & (HO) & - & - & $\ldots$ & 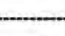 & $\cdots$ & $\cdots$ \\
\hline & 2 & \multicolumn{2}{|c|}{ ppm } & \multicolumn{3}{|c|}{$\mathrm{meq} / 100 \mathrm{~g}$} & $(\%)$ \\
\hline $\begin{array}{l}\text { ARBORIZ. } \\
\text { URBANA }\end{array}$ & 5,6 & 71 & 16 & 1,24 & 0,20 & 0,1 & 1,46 \\
\hline $\begin{array}{l}\text { AREA } \\
\text { CULTIVADA }\end{array}$ & 5,9 & 48 & 5 & 1,86 & 0,48 & 0,2 & 2,13 \\
\hline CAMPINARANA & 3,9 & 2 & 31 & 0,08 & 0,07 & 2,2 & 4,19 \\
\hline CAMPO & 5,7 & 57 & 20 & 0,58 & 0,15 & 0,3 & 1,51 \\
\hline IGAPO & 4,2 & 6 & 43 & 0,15 & 0,16 & 2,9 & 4,25 \\
\hline MATA PAIMARIA & 5,0 & 2 & 21 & 0,14 & 0,11 & 0,5 & 0,62 \\
\hline $\begin{array}{l}\text { MATA } \\
\text { RIBEIRINHA }\end{array}$ & 4,9 & 4 & 51 & 0,62 & 0,65 & 0,9 & 2,90 \\
\hline $\begin{array}{l}\text { MATA } \\
\text { SECUNDÁRIA }\end{array}$ & 4,4 & 7 & 67 & 0.63 & 0,37 & 2,0 & 5,38 \\
\hline SAVANA & 5,4 & 5 & 34 & 0.19 & 0.19 & 0,3 & 0,80 \\
\hline VARZEA & 5.5 & 55 & 226 & 5.61 & 1,49 & 0,3 & 4,87 \\
\hline VAZANTE & 4,1 & 4 & 23 & 0,15 & 0,16 & 2,4 & 5,39 \\
\hline
\end{tabular}

econômica que apresentam.

Com base nas listagens conhecidas, (ALLEN \& ALLEN, 1981; HALLIDAY \& NAKAO, 1982), dois novos gêneros nodulíferos estão sendo reportados neste trabalho: Acosmium (FAB) e Zollernia (CAE). São também apresentadas 21 novas descrições de ocorrência de nodulação em essências indígenas e de 11 espécies que não apresentaram habilidade nodulífera.

\section{CONCLUSÕES}

Foi efetuado um estudo sobre a capacidade de nodulação de 100 espécies amazônicas sendo identificado que $63 \%$ das espécies estão aptas a promover a simbiose com rizóbio. As coletas efetuadas abrangeram 58 gêneros, dos quais 38 deles foram nodulíferos. Esta característica foi observada em 11 diferentes habitats. A nodulação foi constatada e caracterizada no campo e sob condições de enviveiramento, em solos ácidos e de baixa fertilidade. Os nódulos apresentaram uma grande variabilidade de cores e formas, predominando coralóides de cor creme.

Dois novos gêneros nodulíferos são reportados: Acosmium e Zollernia, assim como novas descrições de 21 espécies nodulíferas (Acosmium nitens (Vog.) Yakovl., Andira parviflora Ducke., Cassia mimosoides L., Elizabetha coccinea Benth., Entada polystachya (L.) DC., Galactia jussiaeana Kunth., Hymenolobium petraeum Ducke., Indigofera lespedesioides H.B.K., Inga disticha Benth., Inga thibaudiana DC, Machaerium floribundum Benth., Mimosa debilis H.B.K., Ormosia costulata (Miq.) Kleinh., Ormosia flava (Ducke) Rudd., Phaseolus pilosus H.B.K., Platymiscium paraense Huber, Sclerolobium chrysophyllum Poepp \& Endl., Sclerolobium paraense Huber., Swartzia laurifolia Benth., Swartzia laxiflora Bong ex Benth., Zollernia paraensis Huber.,) e 11 novas 
observações sobre espécies cuja capacidade de formar nódulos não foi constatada (Batesia floribunda Spr. ex Benth., Bauhinia angularis Harms., Bauhinia purpurea DC ex Walp., Bauhinia ungulata L., Cassia lucens Vog., Coursetia ferruginea (Kunt.) Lavin., Cynometra sprucenana var. phaselocarpa (Hayne) Dwyer., Eperua duckeana Cowan., Eperua glabriflora (Ducke) Cowan., Inga myriantha Poepp., e Peltogyne gracilipes Ducke.).

O habitat de ocorrência das espécies, hábito de crescimento, textura e baixa fertilidade dos solos, não interferiram no estabelecimento da nodulação, que está condicionada à outros fatores como a propriedade intrínseca das espécies, e ocorrência de rizóbio compatível em cada local.

\section{AGRADECIMENTOS}

Agradecemos ao Projeto Amazônia I (INPA/Centro de Energia Nuclear na Agricultura/International Atomic Energy Agency) e ao Projeto Maracá (INPA/ Royal Geographycal Society), pelo apoio financeiro.

\section{Bibligrafia citada}

ALLEN, O.N.; ALLEN, E.K. 1981. The leguminosae: A source book of characteristics, uses and nodulation. The University of Wisconsin Press, 812 p.

CARVALHO, M.M.; EDWARDS, D.G.; ASHER, C.J.; ANDREW, C.S. 1982. Effect of aluminium on nodulation of two Stylosanthes species grown in nutrient solution. Plant and Soil, 64: 141- 152.

DUCKE, A. 1949. As leguminosas da Amazônia Brasileira. Notas sobre a flora neotrópica. I.A.N., Bol. Técn. No. 18., 249 p.
DUCKE, A.; BLACK, G.A. 1954. Notas sobre a fitogeografia da Amazônia Brasileira. I.A.N., Bol. técn. $N^{\circ}, 29,38 \mathrm{p}$.

EMBRAPA 1979. Manual de métodos de análise de solo serviço nacional de levantamento $\mathrm{e}$ conservação de solos. Rio de Janeiro, 98p.

FARIA, S.M; FRANCO, A.A.; JESUS, R.M.; MENANDRO, M.S.; BAITELLO, J.B.; MUCCI, E.S.F; DOBEREINER, J.; SPRENT, J.I. 1984. New nodulating legume trees form south-east Brasil. New Phytol., 98:317-328.

HALLIDAY, J.; NAKAO, P.L. 1982. The symbiotic affinities of woody species under consideration as nitrogen-fixing trees. Niftal Project. University of Hawaii, 85p.

LOUREIRO, A.A.; SILVA, M.F; ALENCAR, J.C. 1979. Essências madeireiras da Amazônia. INPA/SUFRAMA, 187p.

MAGALHÃES, FM.M.; MAGALHĀES, L.M.S.; OLIVEIRA, L.A.; DOBE-REINER, J. 1982. Ocorrência de nodulação em leguminosas florestais de terra firme da Região de Manaus.Acta Amazonica, 12 (3): 509-514.

MAGALHĀES, F.M.M.; SILVA, M.F. 1986/87. Associações Rhizobium-leguminosas no estado de Rondônia. Acta Amazônica, 16/ 17:7-17.

MAGALHÃES, L.M.S. 1983. Avaliação edáficonutricional de plantios expe-rimentais de três espécies florestais, em diferentes solos, na regiāo de Manaus-AM. Tese de Mestrado INPA/FUA, 94p.

MATOS, A.O. 1986. Ocorrência de nodulação espontânea em leguminosas florestais nativas de Capitão-poço-PA. Simp.Trop. åmido, I. Anais... Vol. I, Clima Solo, Belém, EMBRAPA, CPATU, 287-294.

MOREIRA, F.M.M.; SILVA, M.F. \& FARIA, S.M. 1992. Occurrence of nodulation in legume species in the Amazon region Brazil. New Phytol., 121: 563-570.

NAS, 1979. Tropical legumes: resources for the future. Washington DC, National Academy of Science, $331 \mathrm{p}$.

NORRIS, D.O. 1969. Observations on the nodulation status of rainforest leguminous species 
in Amazonia and Guyana. Trop. Agric., Trinidad, 46:145-151.

PRANCE, G.T. 1975. Estudos sobre a vegetaçâo de campinas amazonicas I - Introdução a uma série de publicações sobre a vegetação de campinas ama-zônicas. Acta Amazônica, 5(3): 207-209.

RUSSO, R.O. 1983. Fijacion de nitrogeno em sistemas agrofloresles via arboles de uso multiple. CATIE, Turrialba, $11 \mathrm{p}$.

SILVA, M.F; CARREIRA, L.M.M; TAVARES, A.S.; RIBEIRO, I.C.; JARDIM, M.A.G; LOBO, M. G.A. \& OLIVEIRA, J. 1989. As leguminosas da Amazônia Brasileira - Lista Prévia. Acta Bot. Bras., 2 (1): 193-237.
SUDAM/IPT, 1981. Grupamento de espécies tropicais da Amazônia por similâtidade de caracteristicas básicas e por utilização. Belém, SUDAM, 237p.

SYLVESTER-BRADLEY, R.; OLIVEIRA, L.A.; POLDESTÁ FILHO, J.A. JOHN, T.V.S.T. 1980. Nodulation of legumes nitrogenase activity of roots and occurence of nitrogen-fixing Azospirillum spp em representative soils of central amazônia Agroecosistems, 6:249-266. 